\title{
A new conservative-dynamic treatment for the acute ruptured Achilles tendon
}

\author{
Felix Neumayer $\cdot$ Elyazid Mouhsine $\cdot$ Yvan Arlettaz \\ Gérald Gremion · Michael Wettstein · Xavier Crevoisier
}

Received: 6 January 2009 / Published online: 2 April 2009

(C) Springer-Verlag 2009

\begin{abstract}
Introduction There is a trend towards surgical treatment of acute ruptured Achilles tendon. While classical open surgical procedures have been shown to restore good functional capacity, they are potentially associated with significant complications like wound infection and paresthesia. Modern mini-invasive surgical techniques significantly reduce these complications and are also associated with good functional results so that they can be considered as the surgical treatment of choice. Nevertheless, there is still a need for conservative alternative and recent studies report good results with conservative treatment in rigid casts or braces.

Patients/method We report the use of a dynamic ankle brace in the conservative treatment of Achilles tendon rupture in a prospective non-randomised study of 57 consecutive patients. Patients were evaluated at an average follow-up time of 5 years using the modified Leppilahti Ankle Score, and the first 30 patients additionally underwent a clinical examination and muscular testing with a Cybex isokinetic dynamometer at 6 and 12 months.

Results We found good and excellent results in most cases. We observed five complete re-ruptures, almost exclusively in case of poor patient's compliance, two
\end{abstract}

F. Neumayer $\cdot$ E. Mouhsine $\cdot$ Y. Arlettaz .

M. Wettstein $\cdot$ X. Crevoisier

Department of Orthopaedics and Trauma Surgery,

University Hospital (CHUV), Lausanne, Switzerland

G. Gremion

Department of Sports Medicine,

University Hospital (CHUV), Lausanne, Switzerland

X. Crevoisier $(\square)$

CHUV Site Hôpital Orthopédique, Pierre-Decker 4,

1011 Lausanne, Switzerland

e-mail: xavier.crevoisier@chuv.ch partial re-ruptures and one deep venous thrombosis complicated by pulmonary embolism.

Conclusion Although prospective comparison with other modern treatment options is still required, the functional outcome after early ankle mobilisation in a dynamic cast is good enough to ethically propose this method as an alternative to surgical treatment.

Keywords Achilles tendon rupture $\cdot$ Conservative treatment $\cdot$ Functional

\section{Introduction}

The acute rupture of the Achilles tendon occurs most commonly in moderately sportive adults in their thirties and forties, but is also seen in younger athletes [23]. Eventually it occurs also in the elderly athletes. There is an ongoing discussion whether a recently ruptured Achilles tendon is best treated by open or mini-invasive suture or conservatively by a casting or bracing technique. Both surgical and conservative treatments have been reported to obtain good and excellent results in most cases. Meta-analyses have shown wound problems, infection, or paresthesia to be significant complications associated with surgical treatment [14, 31], even though percutaneous techniques seem to reduce the rate of complications [7]. On the other hand, the conservative treatment is more often complicated by re-rupture of the Achilles tendon $[14,16,31]$. Recent surgical and conservative procedures favour a functional bracing to rigid casting $[3,11,19$, 20, 24-26, 29]. To the best of our knowledge, all reports published about conservative treatment were using a rigid bracing type. Our aim was to evaluate a new functional conservative treatment of the recently ruptured Achilles tendon, using an articulated dynamic ankle brace. 


\section{Materials and methods}

Between 1998 and 2005, 57 consecutive patients who attended our outpatient clinic underwent a functional and conservative treatment for an acutely ruptured Achilles tendon. The rupture was diagnosed by a positive Thompson test (calf squeeze test) in all cases, but in four cases confirmation was sought by ultra sound. We included patients of any age and any mechanism of injury with a first episode of an acute rupture of the Achilles tendon (less than 10 days old). The risks and benefits of both surgical and conservative treatments were carefully explained to the patients, who then gave informed consent for this new treatment option. Professional athletes and patients with a re-ruptured Achilles tendons were excluded.

The bracing and rehabilitation procedure was as follows (reproduced also on Table 1): after immobilisation with a cast in equinus for 10 days, the patients were authorised to walk with full weight bearing, protected by a commercial orthosis $\left(\mathrm{VACO}^{\circledR}\right.$ ped, OPED; Fig. 1). The equinus angle was set at $30^{\circ}$ plantar flexion until the end of week 3 , and at $15^{\circ}$ until the end of week 4 . At the fifth week the system was unlocked to allow ankle mobilisation of $30^{\circ}-15^{\circ}-0^{\circ}$, and at $30^{\circ}-0^{\circ}-0^{\circ}$ at the seventh week. The orthosis was removed after 8 weeks. After removal of the orthosis, the patients wore a $10 \mathrm{~mm}$ heel lift for another 4 weeks. Physiotherapy started during the second week with endurance and proprioceptive training with the orthosis in place and active-assisted mobilisation of the tibio-talar joint without the brace. Patients continued physiotherapy for up to 3 months after the accident. Deep vein thrombosis prophylaxis was given for 4 weeks either by low molecular weight heparins or by vitamin $\mathrm{K}$ antagonists.

All patients had follow-up examinations up to 12 months after the trauma. The first 30 patients additionally underwent

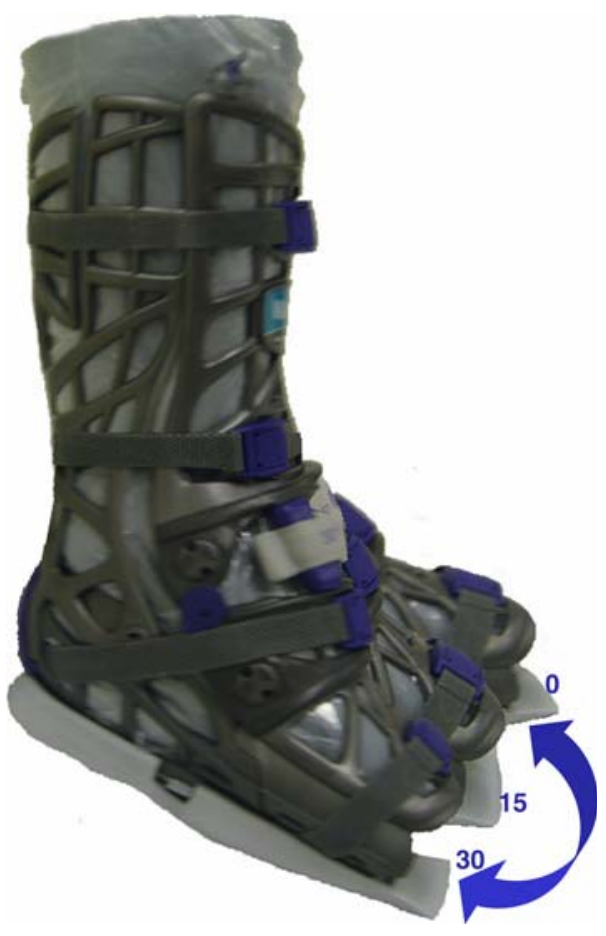

Fig. 1 The orthosis used from day 10 to the eighth week, permitting variable degrees of static or dynamic plantar flexion

muscular testing with a Cybex isokinetic dynamometer at 6 and 12 months. In June 2006 all patients were contacted and received a questionnaire. Subjective opinion of the outcome on a visual analogue scale from 0 to 10 , any change in sporting activities and eventual late complications were investigated. We used a scoring system which was modified from the Leppilahti Ankle Score [17] by van der Lindenvan der Zwaag et al. [18] (Table 2). The Leppilahti Ankle Score has been used by several other authors [21, 29], but has not yet been validated.

Table 1 Re-education and bracing procedure using a dynamic orthosis ( $\mathrm{VACO}^{\circledR}$ ped)

\begin{tabular}{|c|c|c|c|c|c|c|}
\hline & Day $1-10$ & Day 10 to 3 weeks & 4th week & 5 th to 6 th week & 7 th week & $\begin{array}{l}\text { 8th to } \\
12 \text { th week }\end{array}$ \\
\hline Immobilisation/bracing & $\begin{array}{l}\text { Plaster cast in } \\
\text { equinus in } \\
30^{\circ} \text { plantar } \\
\text { flexion }\end{array}$ & $\begin{array}{l}\mathrm{VACO}^{\circledR} \text { ped } \\
\text { static in } \\
30^{\circ} \text { plantar } \\
\text { flexion }\end{array}$ & $\begin{array}{l}\mathrm{VACO}^{\circledR} \text { ped static } \\
\text { in } 15^{\circ} \text { plantar } \\
\text { flexion }\end{array}$ & $\begin{array}{l}\mathrm{VACO}^{\circledR} \text { ped dynamic } \\
\text { in } 15-30^{\circ} \text { plantar } \\
\text { flexion }\end{array}$ & $\begin{array}{l}\mathrm{VACO}^{\circledR} \text { ped dynamic } \\
\text { in } 0-30^{\circ} \text { plantar } \\
\text { flexion }\end{array}$ & $\begin{array}{l}\text { Heel lift } \\
\quad 10 \mathrm{~mm}\end{array}$ \\
\hline Anti-coagulation & \multicolumn{2}{|l|}{$\begin{array}{l}\text { LMWH or } \\
\text { anti-Vitamin K }\end{array}$} & & None & & \\
\hline Weight bearing & None & $\begin{array}{l}\text { As much } \\
\text { as comfortable }\end{array}$ & Full & & & \\
\hline \multicolumn{7}{|l|}{ Physiotherapy } \\
\hline Tibio-talar mobilisation & - & Active-assisted & & Free & & \\
\hline $\begin{array}{l}\text { Proprioception, strength, } \\
\text { endurance }\end{array}$ & - & With orthosis & & & & Free \\
\hline Walking in pool & - & With orthosis & & & & Free \\
\hline
\end{tabular}


Table 2 Modified Leppilahti Ankle Score [23]

\begin{tabular}{lc}
\hline Clinical factor & $\begin{array}{l}\text { Scores } \\
\text { (max. 70 pts) }\end{array}$ \\
\hline Pain & 15 \\
None & 10 \\
Mild, no limited recreational activities & 5 \\
Moderate, limited recreational activities, & \\
$\quad$ but not daily activities & 0 \\
Severe, limited recreational and daily activities & \\
Stiffness & 15 \\
None & 10 \\
Mild, occasional, no limited recreational activities & 5 \\
Moderate, limited recreational activities, & \\
$\quad$ but not daily activities & 0 \\
Severe, limited recreational and daily activities & \\
Calf muscle weakness (subjective) & 15 \\
None & 10 \\
Mild, no limited recreational activities & 5 \\
Moderate, limited recreational activities, & \\
$\quad$ but not daily activities & \\
Severe, limited recreational and daily activities & 0 \\
Foot wear restrictions & \\
None & \\
Mild, most shoes tolerated & \\
Moderate, unable to tolerate fashionable shoes, \\
modified shoes tolerated
\end{tabular}

Modified by van der Linden-van der Zwaag et al. [18]

\section{Results}

Our study group included 12 women and 45 men with an average age of 45 years (24-73). Most of the injuries were due to indirect trauma during sport activities. Soccer, tennis and squash were most frequent but some ruptures happened during long distant running, ski or snowboard activities. Some patients took part in sport up to six times a week, while some did not do any (mean 1.9x/week). Two patients already had a history of Achilles tendopathy on the ruptured side. None had had local infiltrations. No patient was under fluoroquinolone antibiotic treatment. Three patients were under low dose corticoid treatment for obstructive pulmonary diseases. One patient had suffered from previous spontaneous pulmonary embolism.

Dynamometric follow-up evaluation demonstrated that the affected side had regained $70 \%$ speed power, $75 \%$ maximal power, and $70 \%$ endurance power at 6 months when compared to the healthy side. At 12 months these values were $92 \%, 90 \%$, and 90\%, respectively (Fig. 2).

We observed five complete re-ruptures and two partial re-ruptures. The partial re-ruptures were diagnosed by MR imaging as well as three out of the five complete re-ruptures. The two other complete re-ruptures were diagnosed clinically. All re-ruptures happened during the first 5 months after the accident. One re-ruptured tendon was sutured and another patient did not come for follow-up. Three patients with a re-rupture and the partial re-ruptures were treated conservatively by prolonged bracing; all healed with fair to good results. Five out of the seven patients with partial or complete re-ruptures had been showing poor compliance to the treatment protocol or caused the re-rupture when falling over. One patient developed a deep venous thrombosis complicated by pulmonary embolism while he was wearing the orthosis; this patient was already known for a previous spontaneous pulmonary embolism. No relationship was found between the occurrence of re-rupture and the use of low dose corticoid treatment. We observed few minor skin complications as superficial ulcerations or complaints about dry skin.

We were able to collect 46 questionnaires from the 57 patients $(82.5 \%)$. Two patients had died due to non-related causes, nine patients could not be located. The average follow-up time for the questionnaires was 5 years (15 months to 8 years). Two-thirds of the questioned patients had returned to their previous level of sporting activity. Of the remaining third, who had reduced or changed their sporting activity, only half did so because of actual pain or calf weakness, while the other half did so because of apprehension.

The average overall satisfaction with the outcome was $8.1 \pm 2$. The average of the modified Leppilahti Ankle Score was $56.8 \pm 14.9$. For patients without major complications the average overall satisfaction was $8.8 \pm 1.4$ and the modified Leppilahti Ankle Score $60.6 \pm 14.4$.

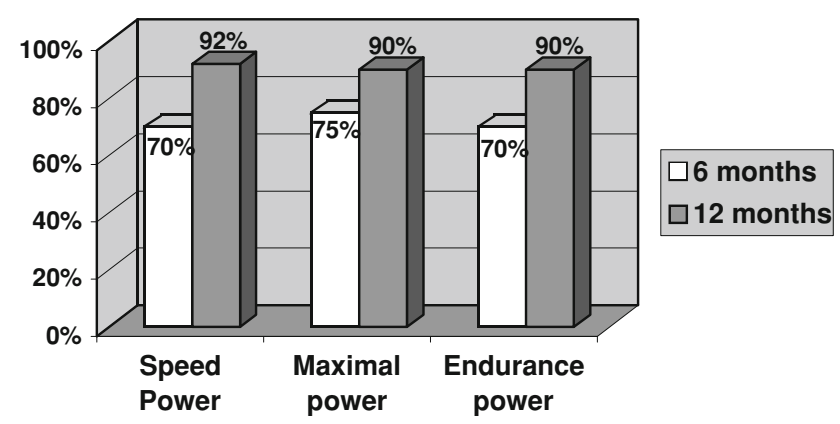

Fig. 2 Dynamometric results in comparison to the healthy side $(=100 \%)$ at 6 and at 12 months after the accident $(N=30)$ 


\section{Discussion}

Post-surgical and conservative "functional" treatment is understood by many authors as a rigid cast or brace immobilisation in full weight bearing. Some allow early ankle mobilisation, taking off the orthosis [11-13, 29, 30]. After an initial period of 2 weeks of below-knee cast immobilisation in full equinus, Saleh et al. [25] were using a splint which immobilised the ankle but allowed movements at the metatarsophalangeal joints. McComis et al. [20] used a brace for a total period of 16 weeks which permitted progressive dorsal extension. When the patient was walking no ankle movement was allowed, but during exercise sessions active ankle flexions were performed. After 4 weeks of rigid immobilisation in gravity equinus, Roberts et al. [24] dynamised their initial below-knee cast by removing a part anteriorly, in order to allow full extension of the ankle. Active ankle flexion was encouraged during exercises, but since the cast stayed rigid it could not enhance ankle movements while walking. While gait analysis has shown the advantage of dynamic bracing in comparison to rigid orthosis in terms of more physiological ambulation [15], to our knowledge, there are no reports published on a conservative treatment of Achilles tendon ruptures with a dynamic orthosis allowing free active ankle movement when walking in full weight bearing.

Dynamic orthosis application, however, has been reported after surgical treatment. In a prospective randomised study, Möller et al. [21] used a functional dynamic brace for their surgically treated patients, while the conservative group was immobilised in a rigid plaster cast. In this study, the surgical treatment was found to be superior, because of a particularly high incidence of re-ruptures in the conservative group $(20.8 \%)$. The operative group had functional rehabilitation, while the conservative treatment consisted of traditional plaster casting; therefore the study does not allow any conclusions about conservative functional treatment of Achilles tendon rupture. On the other hand, it seems to confirm the benefit of early ankle mobilisation and weight bearing for tendon healing. Calder and Saxby [6] were also using a dynamic brace for the postop- erative period in a prospective study of mini-invasive Achilles tendon suturing; they achieved good and excellent results.

It has been demonstrated in animal models that mechanical stress in frequent exercise is an important factor for the quality of tendon healing [8, 9, 22, 28]. This is confirmed by the superior results of functional conservative or postsurgical treatment procedures in comparison to non-weight bearing plaster cast immobilisation [14, 31]. Therefore, it seems logical that early ankle mobilisation in a dynamic cast should promote better functional results than a rigid immobilisation technique.

The mean modified Leppilahti Ankle Score in our study was comparable to two recent studies on conservative treatment procedures who were using the same scoring system [18, 30] (Table 3). Our functional results regarding force and endurance at 12 months were very encouraging. Prospective randomised trials, however, will have to be carried out, to find out if there is a statistically significant difference to functional treatment in rigid orthosis.

According to a cross-sectional study from 2002, after conservative treatment an average of $62 \%$ of patients were able to return to their previous level of sports participation [16]. More recent studies report 49-75.2\% [11, 13, 18]. Our results concur with these reports (Table 3). A high percentage of patients, did not return to their former sports level, because they feared re-rupture. This underlines the necessity, to reassure and accompany the patients until tendon healing has occurred completely and while restarting sporting activities.

Our clinical follow-up period was 12 months for all patients and for the questionnaire the minimum was 18 months. In the meta-analysis of Besch et al. [4], 16 studies were identified who indicated the time interval of their re-ruptures. All appeared during the first 6 months after removing of the cast, only one happened at 9 months due to a new trauma [29]. Therefore, our follow-up time should be sufficient to detect all re-ruptures.

A mean rate of re-rupture of $12.1 \%$ was found in conservative treatment in Kocher's et al. cross-sectional study [16], while newer studies report only $2.1-7 \%[12,13,18$,

Table 3 Comparison of results of recent studies on conservative treatment procedures

\begin{tabular}{|c|c|c|c|c|c|}
\hline & Wallace et al. [30] & $\begin{array}{l}\text { van der Linden-van der der } \\
\text { Zwaag et al. [18] }\end{array}$ & Ingvar et al. [12] & Hufner et al. [11] & Present study \\
\hline Number of patients & 140 & 80 & 196 & 125 & 57 \\
\hline $\begin{array}{l}\text { Return to sport at the } \\
\text { same level }\end{array}$ & $37 \%$ & $49 \%$ & - & $75.2 \%$ & $67.4 \%$ \\
\hline Modified Leppilahti Score & 61.4 & 59 & - & - & 60.6 \\
\hline Re-rupture rate (partial) & $2.1 \%(3.6 \%)$ & $5 \%$ & $7 \%$ & $6.4 \%$ & $9 \%(3.5 \%)$ \\
\hline Other complications & $\begin{array}{l}2 \text { DVT } 1 \text { temporary } \\
\text { dropfoot }\end{array}$ & $1 \mathrm{DVT}+$ pulm. embolism & $\begin{array}{l}7 \text { DVT } 1 \text { pulm. } \\
\text { embolism }\end{array}$ & $3 \mathrm{DVT}$ & $\begin{array}{l}1 \mathrm{DVT}+\text { pulm. } \\
\text { embolism }\end{array}$ \\
\hline
\end{tabular}


29] (Table 3). Hufner et al. [11] pre-selected their patients by ultrasound examinations: inclusion criteria was a complete apposition of the tendon ends in $20^{\circ}$ of plantarflexion. They still reported $6.4 \%$ re-rupture in their conservative functional treatment group. In our study, although we did not apply such strict patients' selection, the rate of re-rupture was only slightly higher (9\%). Furthermore, re-ruptures almost exclusively occurred in patients with poor compliance. Nevertheless, we shall need a larger number of patients to demonstrate that the early ankle mobilisation in full weight bearing in our treatment procedure is not a risk factor for re-rupture.

Functional results of operative and conservative treatment seem to be more or less equivalent [3, 21, 27, 30], although young athletes are thought to benefit more from operative techniques [23]. Risk factors for post-operative complications after Achilles tendon suture are tobacco and steroid use, diabetes and female sex [5]. Especially in patients with more than one risk factor, functional conservative treatment should be the treatment of choice. In patients unable to follow a rehabilitation program or noncompliance, conservative plaster cast immobilisation can be considered, if functional results are of little concern [2].

Classic open surgery for acutely ruptured Achilles tendon seems to progressively disappear from the recent literature. Inversely, there is a trend towards mini-open techniques $[1,3,6,7,10]$ and the interest in conservative treatment has also been renewed, based on recent studies reporting the efficiency of this therapeutic solution [11-13, $18,29]$. Nevertheless, to our knowledge, there is no existing study reporting prospective comparison between these two most recommended treatment options. Both strategies have been recently applied on relatively large numbers of patients and were associated with equivalent good results. We still need prospective randomised controlled studies to compare functional results and gait. We also need to further investigate re-rupture rates and surgical risks and complications. It would be important to analyse if functional and subjective results depend only on the treatment procedure or also on patient age and sports activity. In the meantime we are ethically in the position to propose either of the treatment options to the patient: mini-invasive surgery or conservative functional management.

There are limitations in the present study. Cybex dynamometric testing was no more available at our institution in the second half of the study so that only the first 30 patients could undergo this specific assessment. Nevertheless, we decided to include the further 27 patients since the dynamometric assessment represents only one part of the study. A follow-up rate of $82.5 \%$ at a mean time of 5 years (and up to 8 years) might be considered as low. However, it appears quite acceptable when compared to the rates of $53-74 \%$ reported in other recent studies [11, 13, 18].

\section{Conclusion}

This is the first published report about the use of a dynamic foot and ankle brace for the conservative treatment of acute Achilles tendon rupture. The present treatment resulted in good to excellent functional results in most cases and, therefore, demonstrates that conservative functional treatment in the acute rupture of the Achilles tendon can be ethically proposed as an alternative to surgical treatment in most cases. It requires patient compliance and active participation, as well as a systematic medical follow-up during the first 6 months. The re-rupture rate of $9 \%$ is slightly higher than in recently published series about conservative treatment but, considering that no patients' selection was applied and also that reruptures occurred almost exclusively in non-compliant patients, it is acceptable. Our results are consistent with recent reports that emphasise the advantages of early ankle mobilisation in aiming good functional results.

\section{References}

1. Amlang MH, Christian P, Heinz P, Zwipp H (2005) Percutaneous technique for Achilles tendon repair with the Dresden: instruments and results. Unfallchirurg 108:529-536

2. Arlettaz Y, Chevalley F, Gremion G, Leyvraz PF (1998) Les ruptures fraîches du tendon d'Achille-A propos de 14 cas traités conservativement. Swiss Surg 4:75-81

3. Assal M, Jung M, Stern R, Rippstein P, Delmi M, Hoffmeyer P (2002) Limited open repair of Achilles tendon ruptures: a technique with a new instrument and findings of a prospective multicenter study. J Bone Joint Surg Am 84:161-170

4. Besch S, Peyre M, Rodineau J, Dupre JP (2006) Traitement conservateur des ruptures du tendon d'Achille. J Traumatol Sport 23:12-24

5. Bruggemann NB, Turner NS, Dahm DL, Voll AE, Hoskin TL, Jacofski DJ, Haidukewych GJ (2004) Wound complications after open Achilles tendon repair. An analysis of risk factors. Clin Orthop Relat Res 427:63-66

6. Calder JD, Saxby TS (2005) Early, active rehabilitation following mini-open repair of Achilles tendon rupture: a prospective study. Br J Sports Med 39:857-859

7. Cretnik A, Kosanovic M, Smrkolj V (2005) Percutaneous versus open repair of the ruptured Achilles tendon. A comparative study. Am J Sports Med 33:1369-1379

8. Enwemeka CS, Spielholz NI, Nelson AJ (1988) The effect of early functional activities on experimentally tenotomized Achilles tendons in rats. Am J Phys Med Rehabil 67:264-269

9. Gelbermann RH, Menon J, Gonsalves M, Akeson WH (1980) The effects of mobilization on the vascularization of healing flexor tendons in dogs. Clin Orthop 153:283-289

10. Gorschewsky O, Pitzl M, Putz A, Klakow A, Neumann W (2004) Percutaneous repair of acute Achilles tendon rupture. Foot Ankle Int 25:219-224

11. Hufner TM, Brandes DB, Thermann H, Richter M, Knobloch K, Krettek C (2006) Long-term results after functional nonoperative treatment of Achilles tendon rupture. Foot Ankle Int 27:167-171

12. Ingvar J, Tägil M, Eneroth M (2005) Nonoperative treatment of Achilles tendon rupture. 196 consecutive patients with a 7\% re-rupture rate. Acta Orthop 76:597-601 
13. Josey RA, Marymont JV, Varner KE, Borom A, O'Connor D, Oates JC (2003) Immediate, full weigthbearing cast treatment of acute Achilles tendon ruptures: a long-term follow-up study. Foot Ankle Int 24:775-779

14. Khan RJK, Fick D, Keogh A, Crawford J, Brammar T, Parker M (2005) Treatment of acute Achilles tendon ruptures. A metaanalysis of randomised, controlled trials. J Bone Joint Surg Am 87:2202-2210

15. Kitaoka HB, Crevoisier XM, Harbst K, Hansen D, Kotajarvi B, Kaufman K (2006) The effect of custom-made braces for the ankle and the foot kinematics and ground reaction forces. Arch Phys Med Rehabil 87:130-135

16. Kocher MS, Bishop J, Marshall R, Briggs KK, Hawkins RJ (2002) Operative versus nonoperative management of acute achilles tendon rupture. Expected-value decision analysis. Am J Sorts Med 30:783-788

17. Leppilahti J, Forsman K, Puranen J, Orava S (1998) Outcome and prognostic factors of Achilles rupture repair using a new scoring method. Clin Orthop Relat Res 346:152-161

18. van der Linden-van der Zwaag HMJ, Nelissen RGHH, Sintenie JB (2004) Results of surgical versus non-surgical treatment of Achilles tendon rupture. Int Orthop 28:370-373

19. Maffulli N, Tallon C, Wong J, Lim KP, Bleakney R (2003) Early weightbearing and ankle mobilization after open repair of acute midsubstance tears of the Achilles tendon. Am J Sports Med 31:692-700

20. McComis GP, Nawoczenski DA, DeHaven KE (1997) Functional bracing for rupture of the Achilles tendon. Clinical results and analysis of ground-reaction forces and temporal data. J Bone Joint Surg Am 79:1799-1808

21. Möller M, Movin T, Granhed H, Lind K, Faxén E, Karlsson J (2001) Acute rupture of tendo Achillis. A prospective, randomised study of comparison between surgical and non-surgical treatment. J Bone Joint Surg Br 83:843-848
22. Palmes D, Spiegel HU, Schneider TO, Langer M, Stratmann U, Budny T, Probst A (2002) Achilles tendon healing: long-term biomechanical effects of postoperative mobilization and immobilisation in a new mouse model. J Orthop Res 20:939-946

23. Rettig AC, Liotta FJ, Klootwyk TE, Porter DA, Mieling P (2005) Potential risk of rerupture in primary Achilles tendon repair in athletes younger than 30 years of age. Am J Sports Med 30:119-123

24. Roberts CP, Palmer S, Vince A, Deliss LJ (2001) Dynamised cast management of Achilles tendon ruptures. Injury 32:423-426

25. Saleh M, Marshall PD, Senior R, MacFarlane A (1992) The Sheffield splint for controlled early mobilisation after rupture of the calcaneal tendon. A prospective, randomised comparison with plaster treatment. J Bone Joint Surg Br 74:206-209

26. Suchak AA, Spooner C, Reid DC, Jomha NM (2006) Postoperative rehabilitation protocols for Achilles tendon ruptures: a metaanalysis. Clin Orthop Relat Res 445:216-221

27. Thermann H, Zwipp H, Tscherne H (1995) Funktionelles Behandlungskonzept der frischen Achillessehnenruptur. Zweijahresergebnisse einer prospektiv-randomisierten Studie. Unfallchirurg 98:21-32

28. Tipton CM, Matthes RD, Maynard JA, Carey RA (1975) The influence of physical activity on ligaments and Tendons. Med Sci Sports 7:165-175

29. Wallace RGH, Traynor IER, Kernohan WG, Eames MHA (2004) Combined conservative and orthotic management of acute ruptures of the Achilles tendon. J Bone Joint Surg Am 86:1198-1202

30. Weber M, Niemann M, Lanz R, Müller T (2003) Nonoperative treatment of acute rupture of the Achilles tendon. Results of a new protocol and comparison with operative treatment. Am J Sports Med 31:685-691

31. Wong J, Barrass V, Maffulli N (2002) Quantitative review of operative and nonoperative management of Achilles tendon ruptures. Am J Sports Med 30:565-575 